Supporting Information for

\title{
Photo-Curable Hyperbranched Polymer Medical Glue for Water-Resistant Bonding
}

Yifan Zhang, Xiaojie Li*, Qinfu Zhu, Wei Wei, and Xiaoya Liu*

Key Laboratory of Synthetic and Biological Colloids, Ministry of Education, School of Chemical and Material Engineering, Jiangnan University, No 1800 Lihu Avenue, Wuxi, Jiangsu, 214122, P. R. China.

*Correspondence to: xjli@jiangnan.edu.cn,lxy@jiangnan.edu.cn 


\section{Synthesis of 2-(acryloyloxy) ethyl methacrylate}

Acrylic acid (144.16 g, $2 \mathrm{~mol})$, phloroglucinol (0.260 g, $20 \mathrm{mmol})$, hydroxyethyl methacrylate (130.14 g, $1 \mathrm{mmol})$ and p-toluenesulfonic acid (5.17 g, $30 \mathrm{mmol})$ were dissolved in toluene $(200 \mathrm{~mL})$, then was heated to $90{ }^{\circ} \mathrm{C}$ for $3 \mathrm{~h}$. Subsequently, the reaction mixture was washed 6 times with deionized water to remove excess reactant, and then dried by anhydrous sodium sulfate. The residue was further purified by a neutral alumina column and then the organic solvent was thoroughly evaporated to afford colorless liquid (125.31 g, 68.6\% yield).

\section{Absolute molecular weight and its distribution:}

The detailed polymer parameters of HBPTE were measured using a multi-angle laser light scattering system (DAWN HELEOS II System, Wyatt Technology Corporation) combine with a gel permeation chromatogram (GPC, Waters 1515-2414-2707, Agilent organic column, PMMA standard, mixed-C column). The DMF added with $10 \mathrm{mmol} \cdot \mathrm{L}^{-1}$ of $\mathrm{LiBr}$ was used as

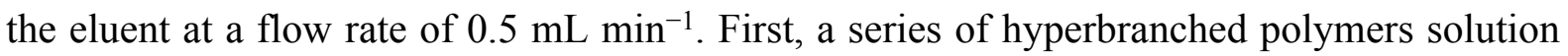
with gradient concentration $(5 \mathrm{mg} / \mathrm{mL}, 10 \mathrm{mg} / \mathrm{mL}, 15 \mathrm{mg} / \mathrm{mL}, 20 \mathrm{mg} / \mathrm{mL}$ and $25 \mathrm{mg} / \mathrm{mL}$ ) were measured though a laser detector $(120 \mathrm{~mW}, \lambda=664 \mathrm{~nm}$, WYATT-1486-H2, DAWN HELEOS) to obtain the $d_{n} / d_{c}$ (specific refractive index increase). Then the intrinsic viscosity of the polymer was detected with a viscosity detector. The original data is processed with Astra VI software, the absolute weight average molecular weight of the sample was fitted by $d_{n} / d_{c}$, and the MHS index $(\alpha)$ was fitted by the intrinsic viscosity and absolute weight average molecular weight.

\section{Rheological properties:}

The viscosity, elastic modulus and loss modulus of HBPTE was determined using a 
Discovery Hybrid Rheometer (DHR-2, TA Instruments, DE, USA) equipped with a parallel plate ( $8 \mathrm{~mm}$ diameter). The gap value was set to $100 \mu \mathrm{m}$ at $25^{\circ} \mathrm{C}$. Before measurement of storage modulus $\left(\mathrm{G}^{\prime}\right)$ and loss modulus $\left(\mathrm{G}^{\prime \prime}\right)$, the linear viscoelastic region (LVR) was determined after a strain-sweep test between $0.1 \%$ and $20 \%$ at a constant frequency of $1 \mathrm{~Hz}$. The LVR was concluded to be around $1 \%$ strain, since $\mathrm{G}^{\prime}$ and $\mathrm{G}^{\prime \prime}$ were linearly dependent with strain around that value. Next, $G^{\prime}$ and $G^{\prime \prime}$ was measured during a dynamic frequency-sweep test in a range of frequencies between $0.1 \mathrm{~Hz}$ and $1 \mathrm{~Hz}$ with a constant strain of $1 \%$ at $25{ }^{\circ} \mathrm{C}$. Then, polymer viscosity was measured as a function of shear rate between 0.1 and $100 \mathrm{~s}^{-1}$.

\section{Transmittance test:}

HBPTE, PEGDMA and $0.2 \mathrm{wt} \%$ Irgacure 2959 was mixed in a quartz cuvette, and then was cured for 1 minute. The transmittance was measured using ultraviolet-visible spectroscopy spectrophotometer (UV-vis, TU-1901) in a range of wavelength between $400 \mathrm{~nm}$ and $900 \mathrm{~nm}$ with an empty cuvette as a reference.

\section{Storage stability:}

Taking HBPTE1-0.2 as an example to evaluate the storage stability of glue by a vial tilting method. The vial contained about $2 \mathrm{~mL}$ of glue was placed in a dark environment at $25^{\circ} \mathrm{C}$, and the time when flow is not observed after tilting was recorded.

\section{Lap shear adhesive test:}

The HBPTE-glue was applied to a glass slide in an area of $2.50 \mathrm{~cm} \times 2 \mathrm{~cm}$. Then, another glass slide was used to overlap over the area with glue applied. Subsequently, the lap joints were cured using UV light irradiation of $10 \mathrm{~J} \cdot \mathrm{cm}^{-2}$ and then immersed into a PBS solution at $37^{\circ} \mathrm{C}$ for two weeks. To prevent breakage of the brittle glass slide with tension applied, two 
iron steel rods were coated with cyanoacrylate glue and superimposed on the slide. Afterwards, lap shear tests were carried out using a universal testing machine (ITW 5967X, USA) equipped with a $500 \mathrm{~N}$ load cell at a tensile speed of $5 \mathrm{~mm} / \mathrm{min}$. The results were reported as average and standard deviations of five measurements.

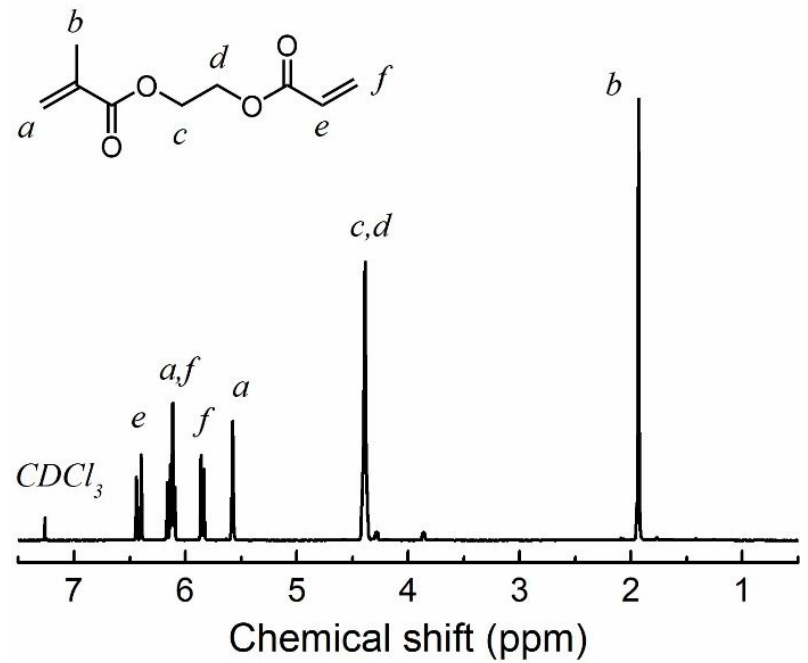

Figure S1: ${ }^{1} \mathrm{H}$ NMR spectrum recorded of 2-(acryloyloxy) ethyl methacrylate in $\mathrm{CDCl}_{3}$.

(A)

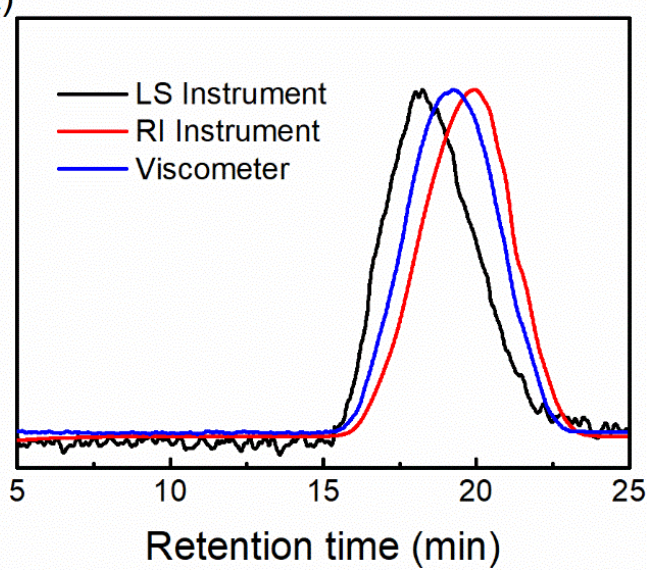

(B)

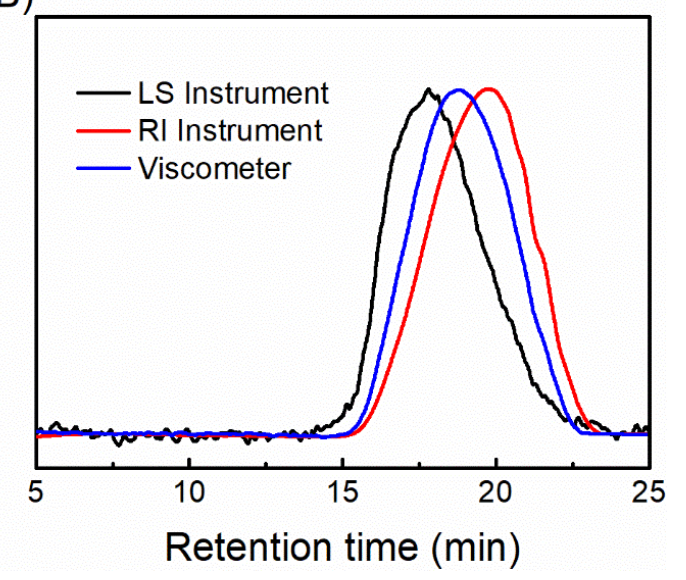

Figure S2: The molecular weight distribution curves of HBPTE1(A) and HBPTE12 (B) recorded by light scattering (LS) detector, refractive index (RI) and viscometer, respectively. 


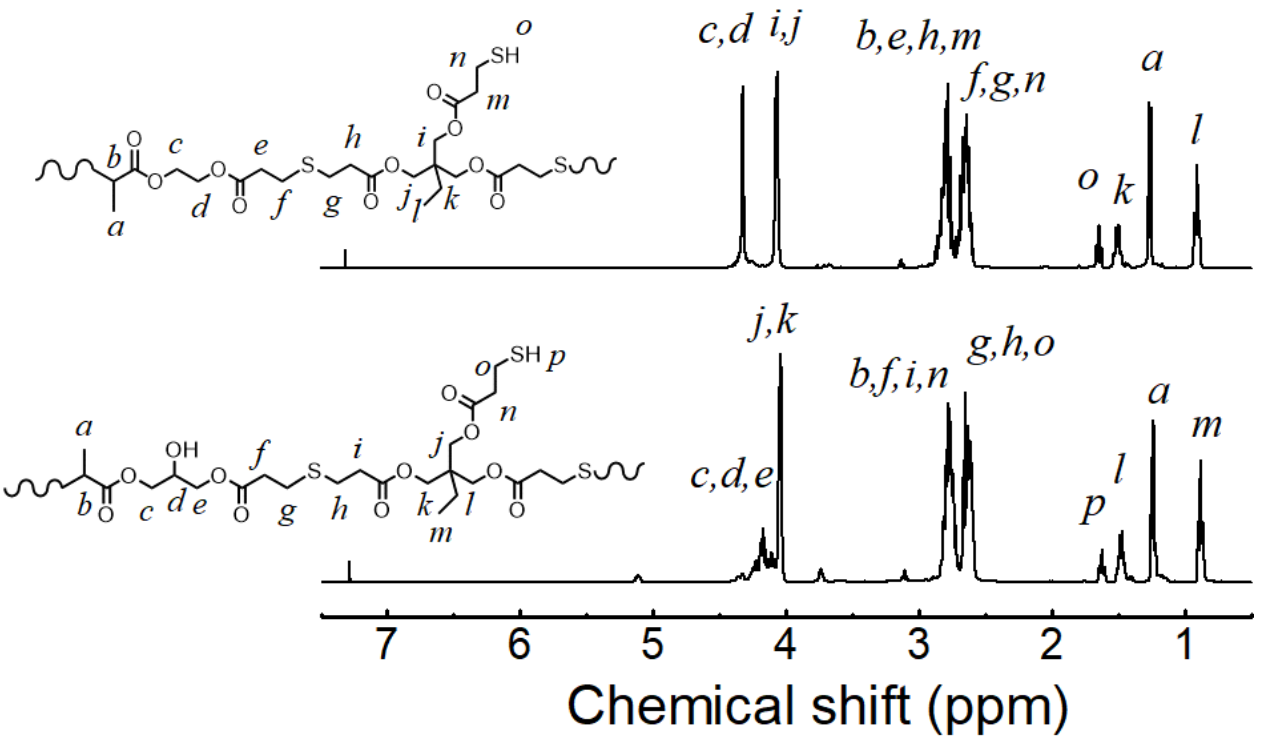

Figure S3: ${ }^{1} \mathrm{H}$ NMR spectra recorded of $\mathrm{HBPTE}$ in $\mathrm{CDCl}_{3}$.
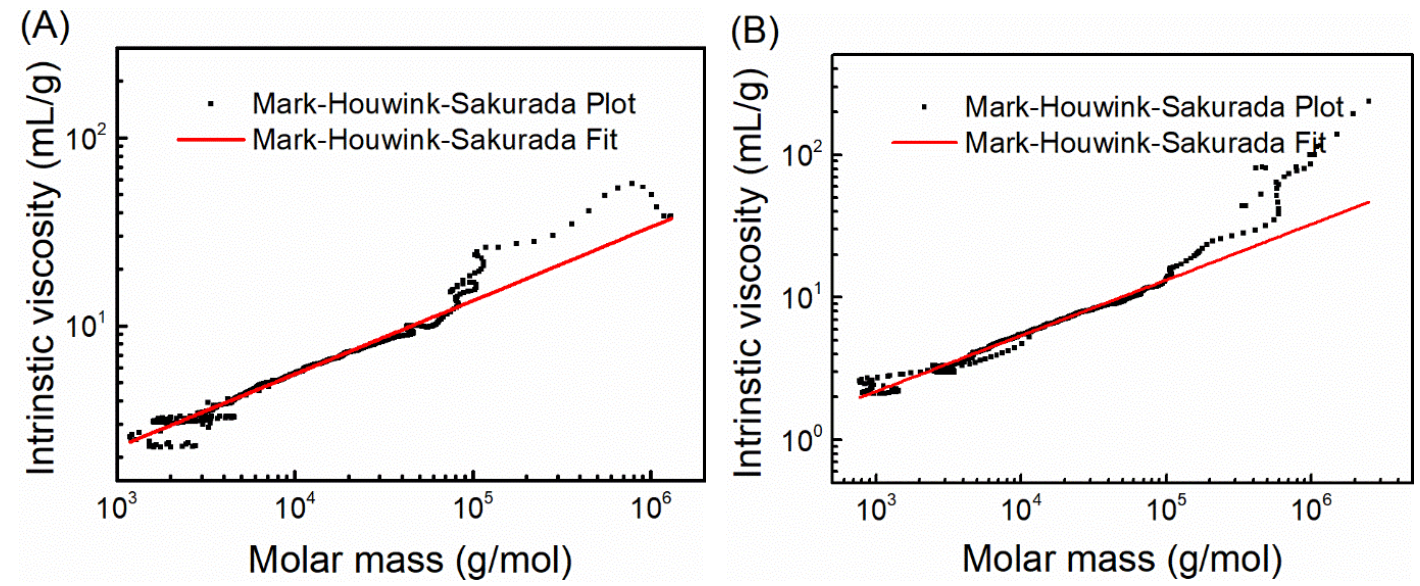

Figure S4: The Mark-Houwink-Sakurada plot and fit line of HBPTE1(A) and HBPTE12 (B).

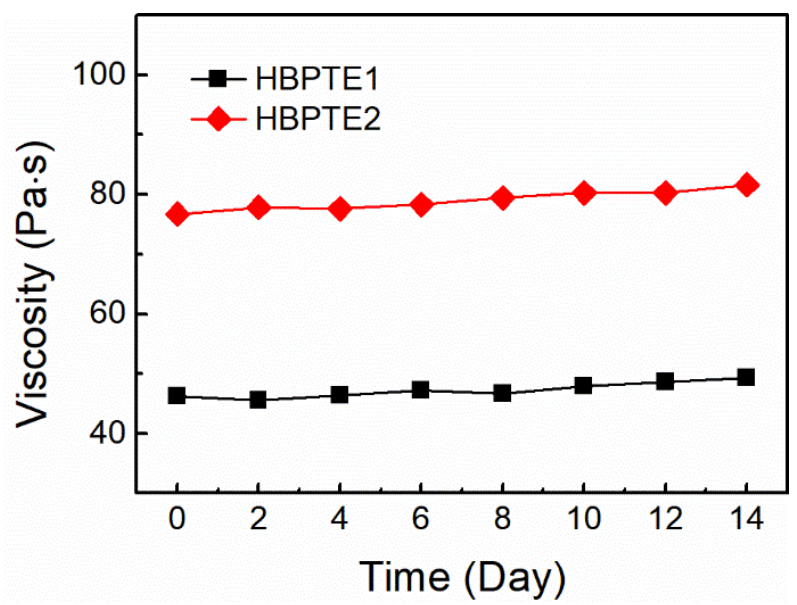

Figure S5: The viscosity change of HBPTE as a function of storage time at room 
temperature.

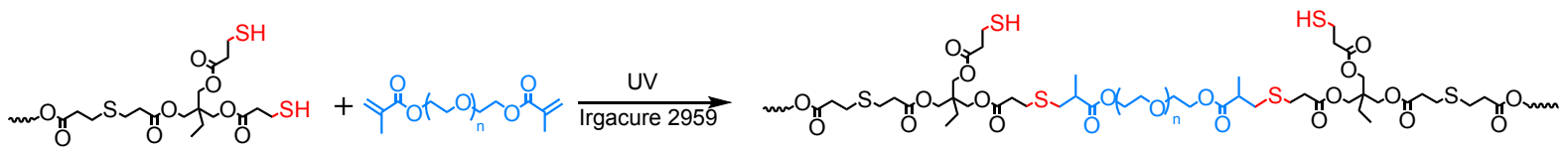

Figure S6: Schematic illustration of photo-crosslinking of HBPTE glue.

(A)

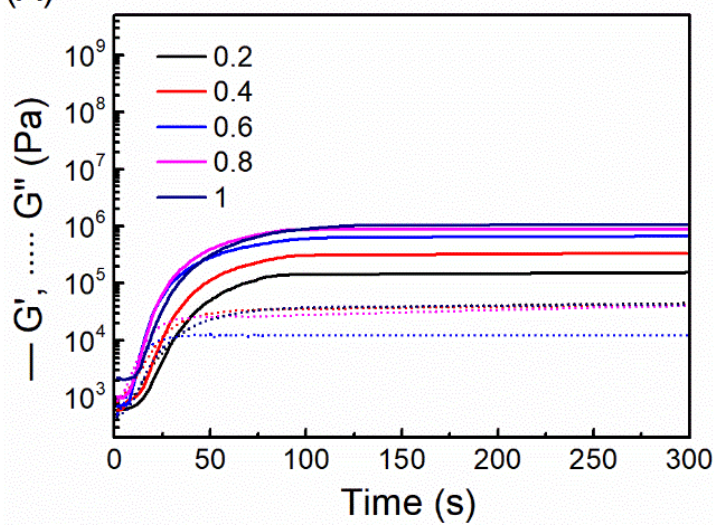

(B)

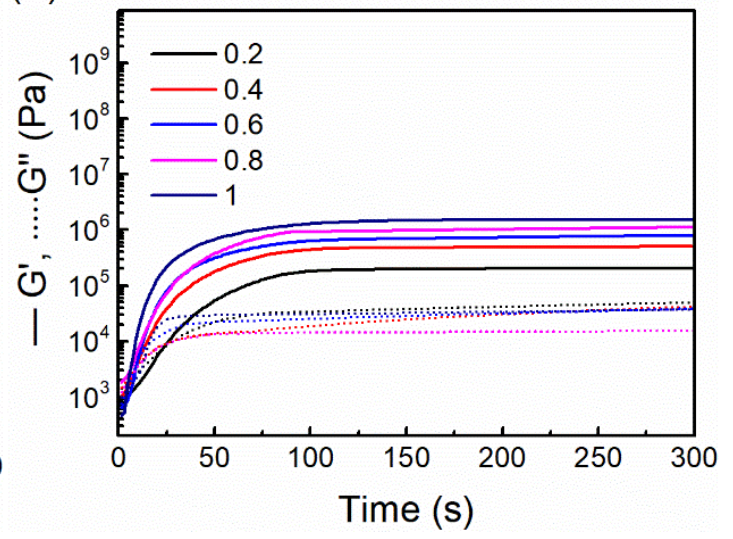

Figure S7: Real time rheology profile of HBPTE1 glue (A) and of HBPTE2 glue (B) at a UV $(365 \mathrm{~nm})$ energy of $20 \mathrm{~mW} / \mathrm{cm}^{2}$.
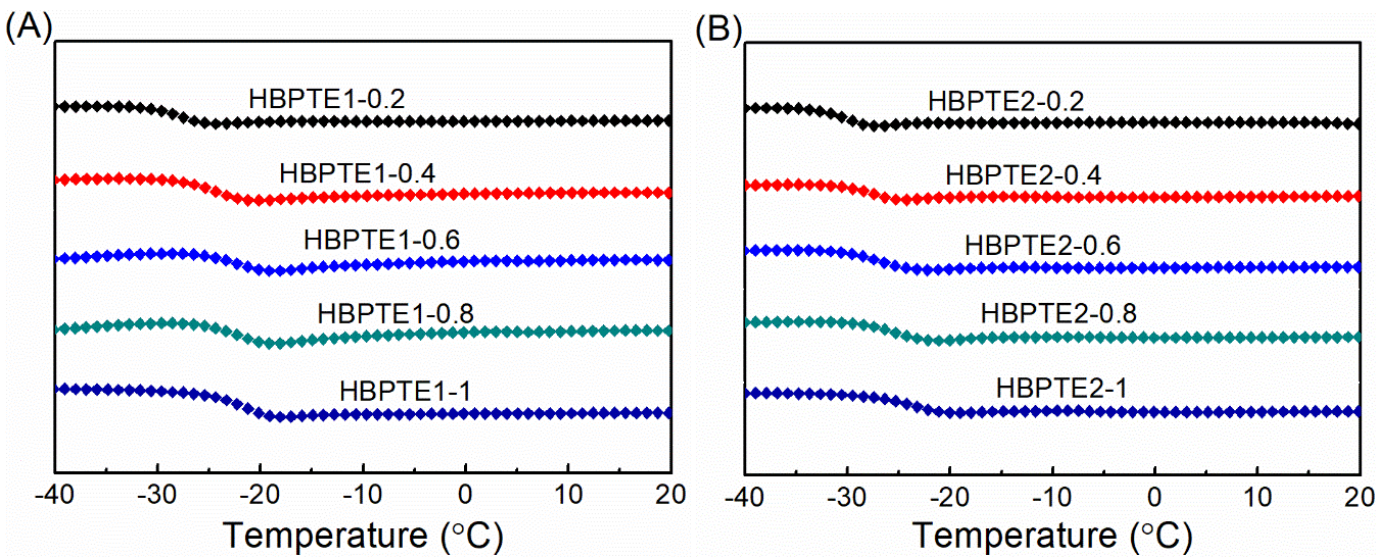

Figure S8: DSC curve of cured HBPTE1 glue (A) and HBPTE2 glue (B). 

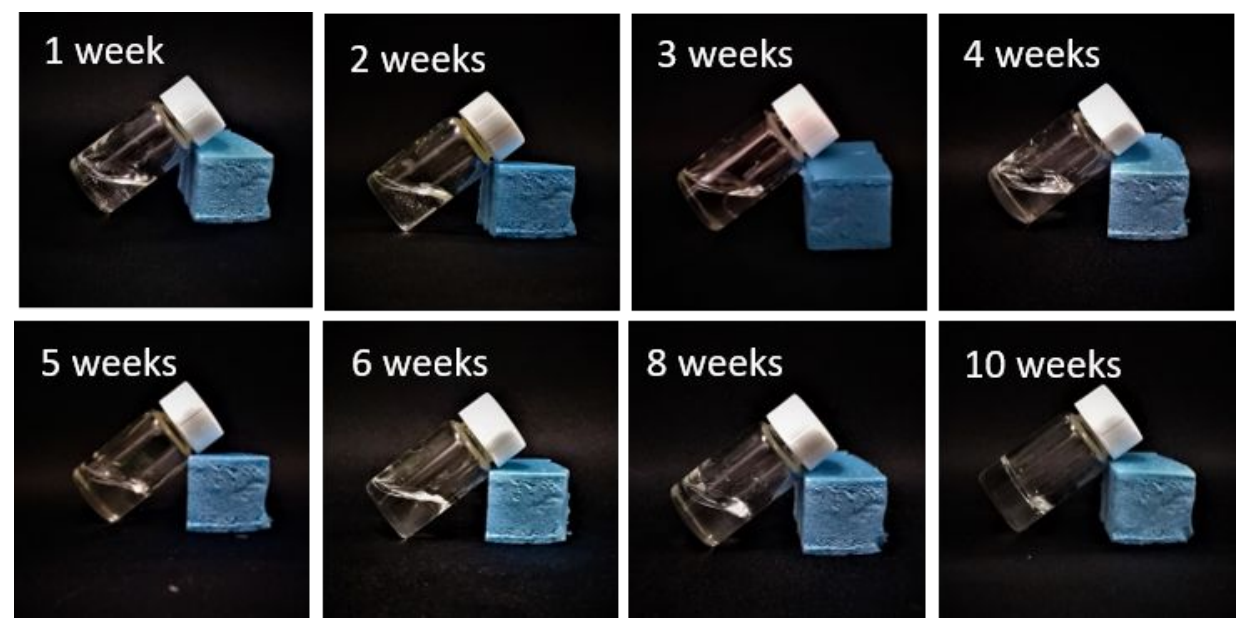

Figure S9: Storage stability test of HBPTE1-0.2 via vial tilting method under dark environment at room temperature.
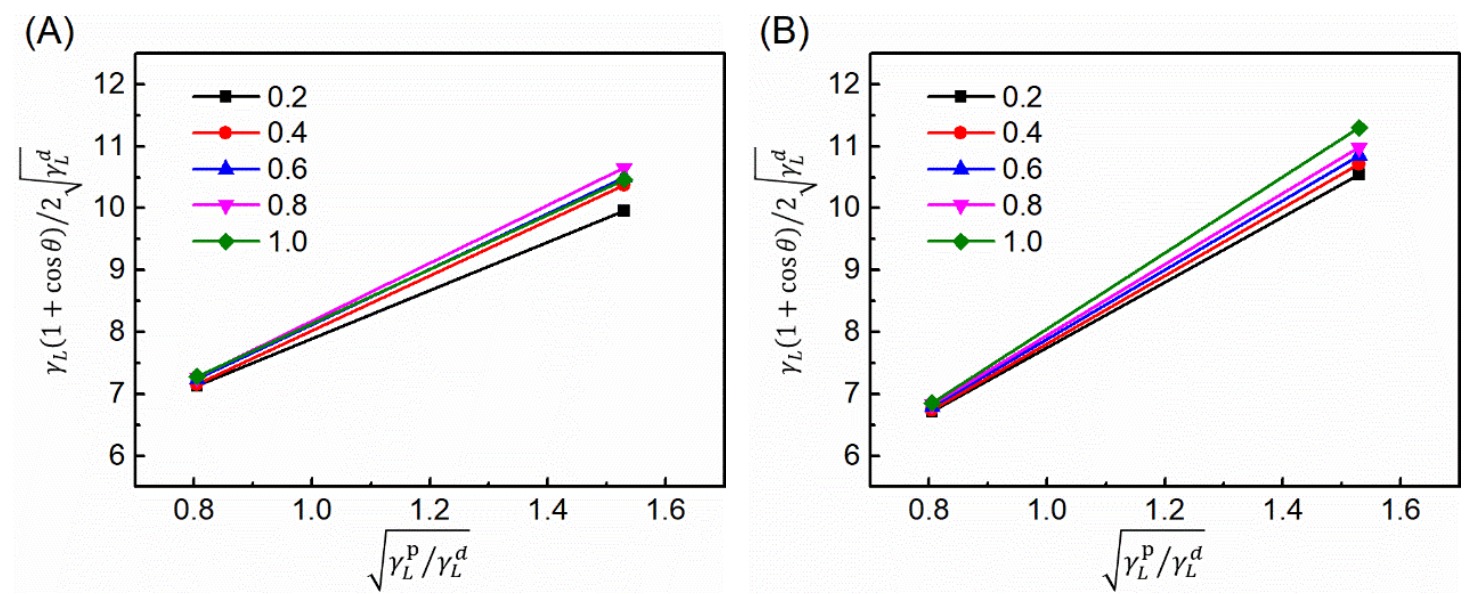

Figure S10: Surface energy fitting plot of HBPTE1 glue (A) and HBPTE1 glue (B) according to the contact angles made by ethylene glycol and water.

Table S1 Summary of the surface energy of HBPTE1 and HBPTE2 glue.

\begin{tabular}{|c|c|c|c|c|c|c|c|c|c|c|}
\hline \multirow{2}{*}{ Sample } & \multicolumn{5}{|c|}{ HBPTE1 glue } & \multicolumn{5}{|c|}{ HBPTE2 glue } \\
\hline & 0.2 & 0.4 & 0.6 & 0.8 & 1 & 0.2 & 0.4 & 0.6 & 0.8 & 1 \\
\hline$\gamma^{d}$ & 15.85 & 12.86 & 13.14 & 12.21 & 11.64 & 6.04 & 5.37 & 5.12 & 4.93 & 3.64 \\
\hline$\gamma^{\mathbf{p}}$ & 15.27 & 19.69 & 20.21 & 21.91 & 23.09 & 27.91 & 30.16 & 31.56 & 32.79 & 37.7 \\
\hline$\gamma$ & 31.12 & 32.55 & 33.35 & 34.12 & 34.73 & 33.96 & 35.53 & 36.68 & 37.72 & 41.34 \\
\hline
\end{tabular}

Polar component $\left(\boldsymbol{\gamma}^{\mathbf{d}}\right)$ and dispersion component $\left(\boldsymbol{\gamma}^{\mathbf{p}}\right)$ of surface energy of HBPTE glue. 


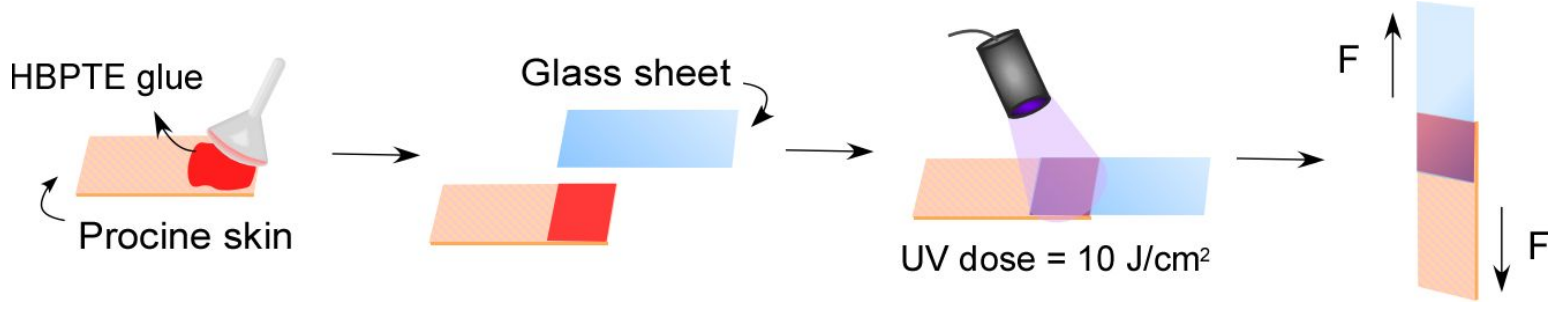

Figure S11: Schematic representation of the lap-shear joints for the porcine adhesive test.

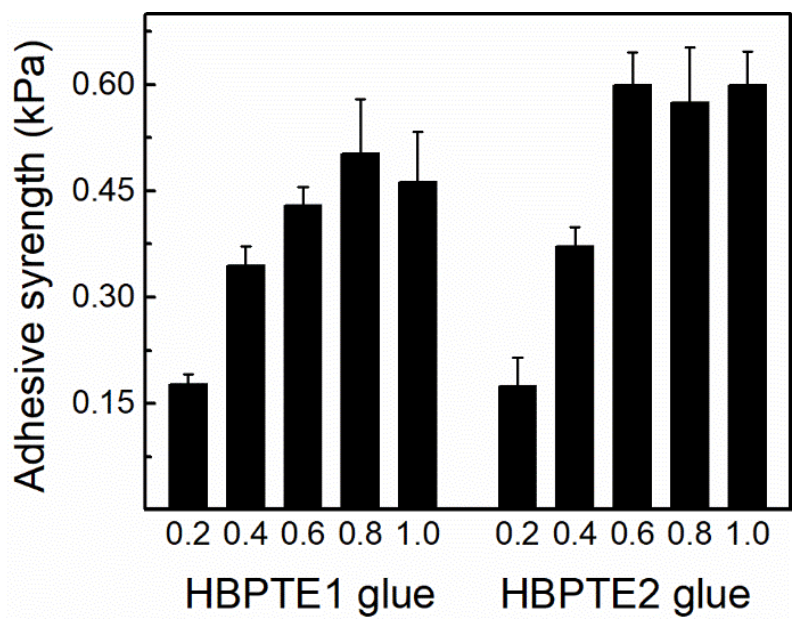

Figure S12: Lap-shear strength of HBPTE glue on glass sheet.

(A)

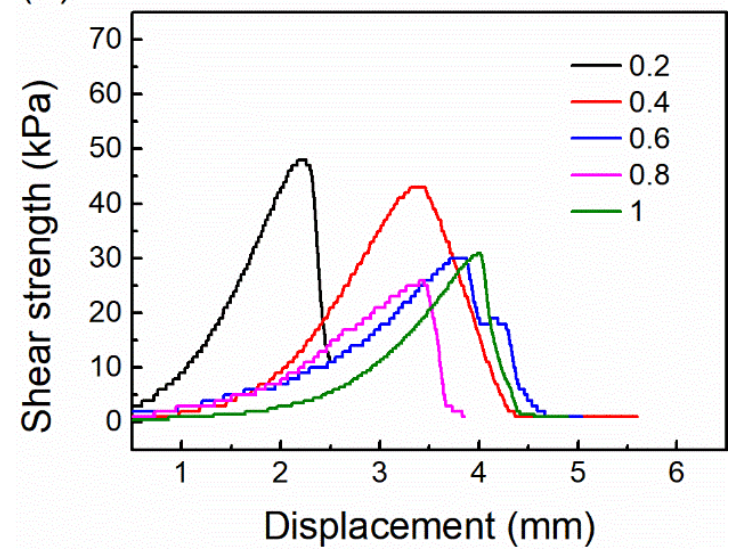

(B)

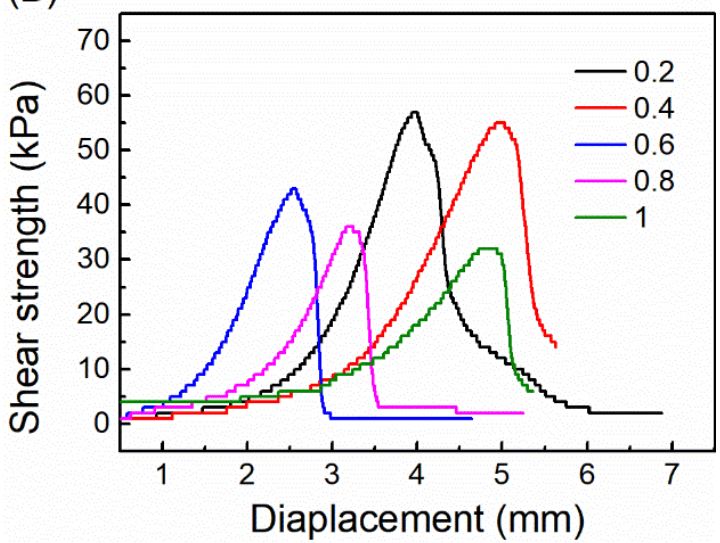

Figure S13: Lap shear bonding curve of the porcine skin sample bonded by HBPTE1 glue (A) and HBPTE2 glue (B). 

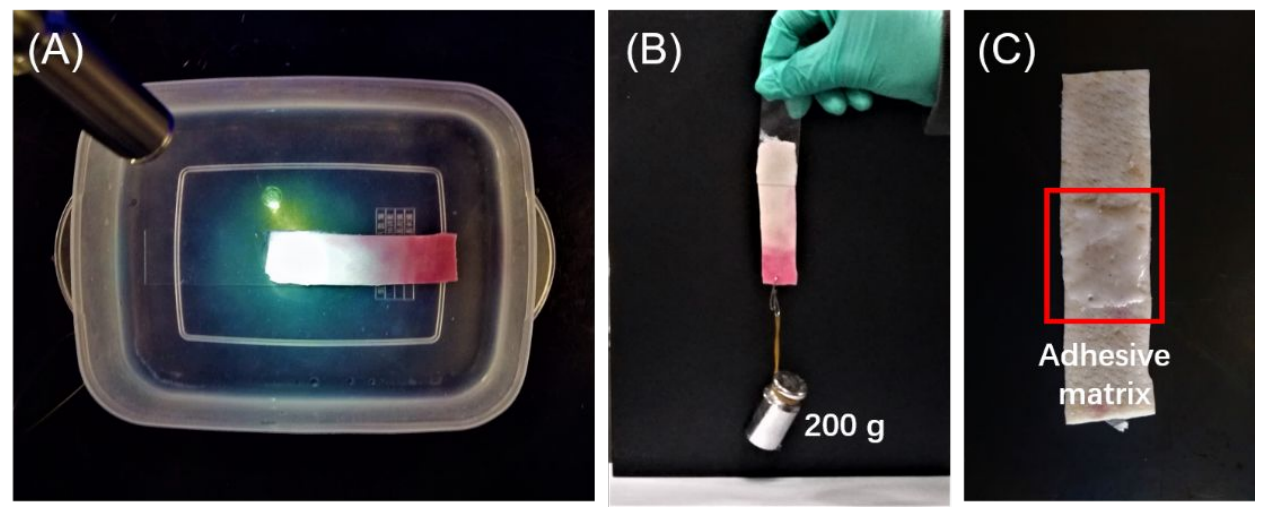

Figure S14: (A) The photo image of underwater adhesion test of HBPTE1-0.2 in PBS (pH =

7.4). (B) The HBPTE1-0.2 bonded porcine can lift a $200 \mathrm{~g}$ weight. (C) The photo image depicting HBPTE1-0.2 still adhered on porcine skin after soaking in water for two weeks.

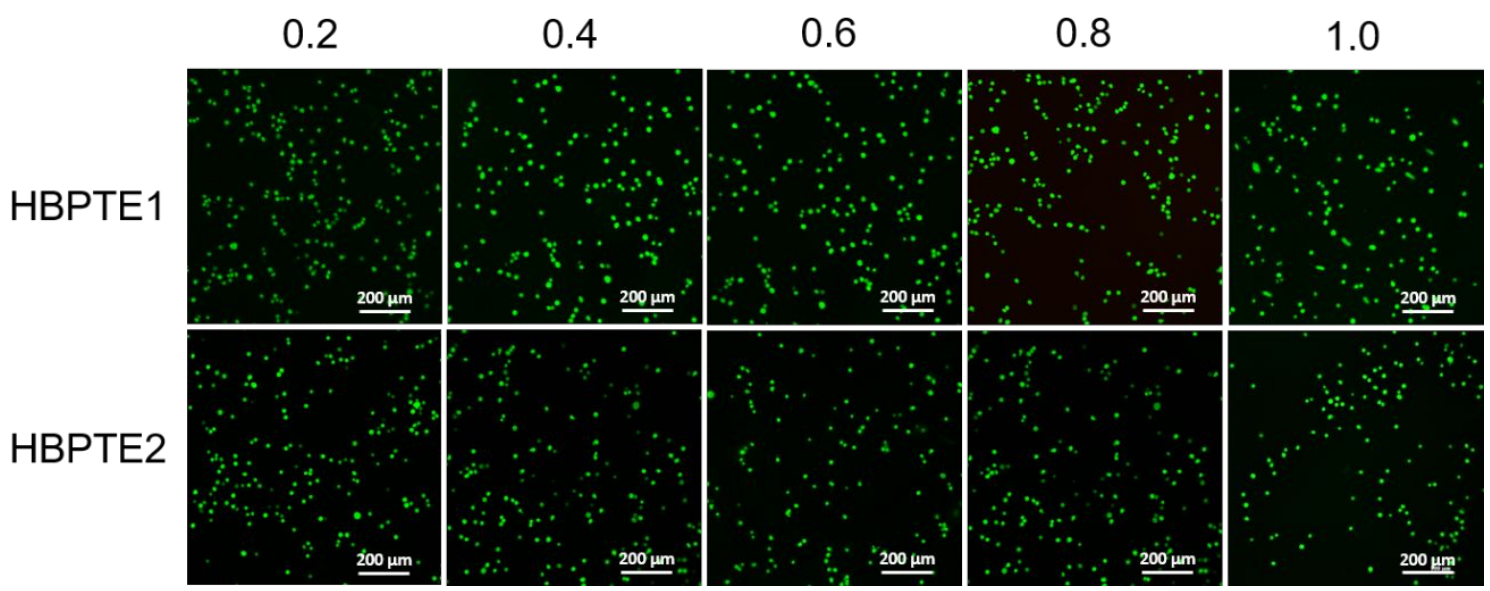

Figure S15: Live/dead staining confocal images of L929 cell present on cured HBPTE glue after $24 \mathrm{~h}$.

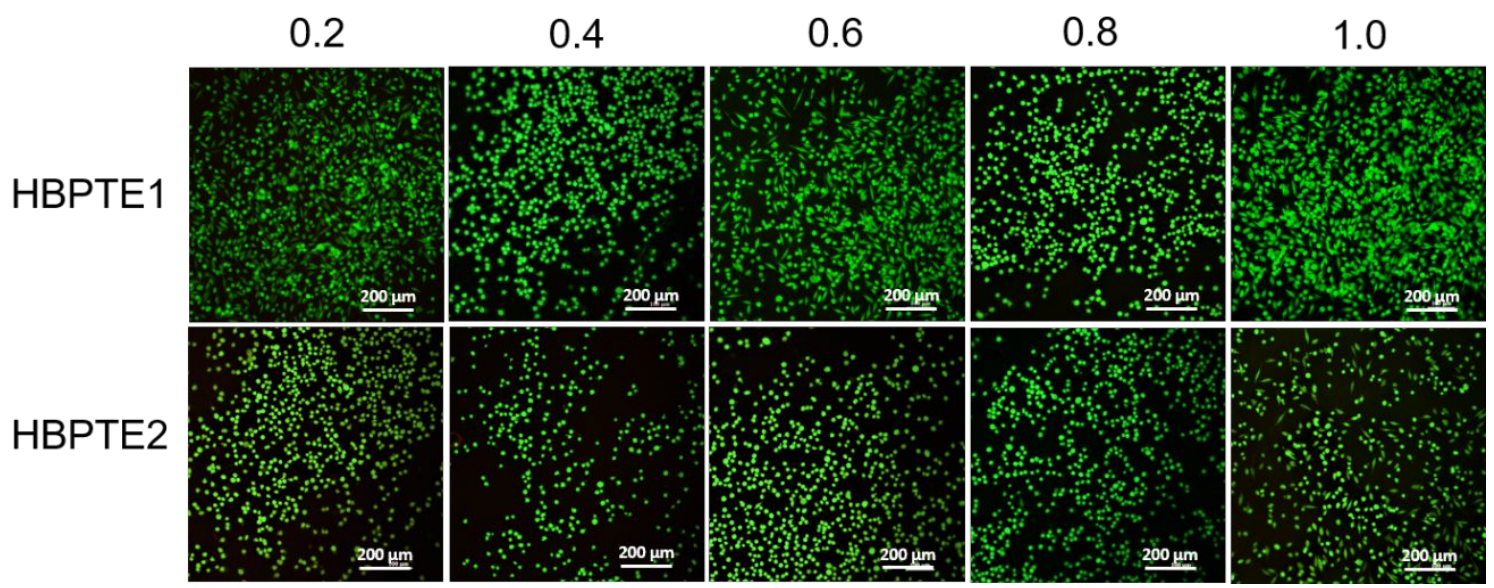

Figure S16: Live/dead staining confocal images of L929 cell present on cured HBPTE glue 
after $48 \mathrm{~h}$.
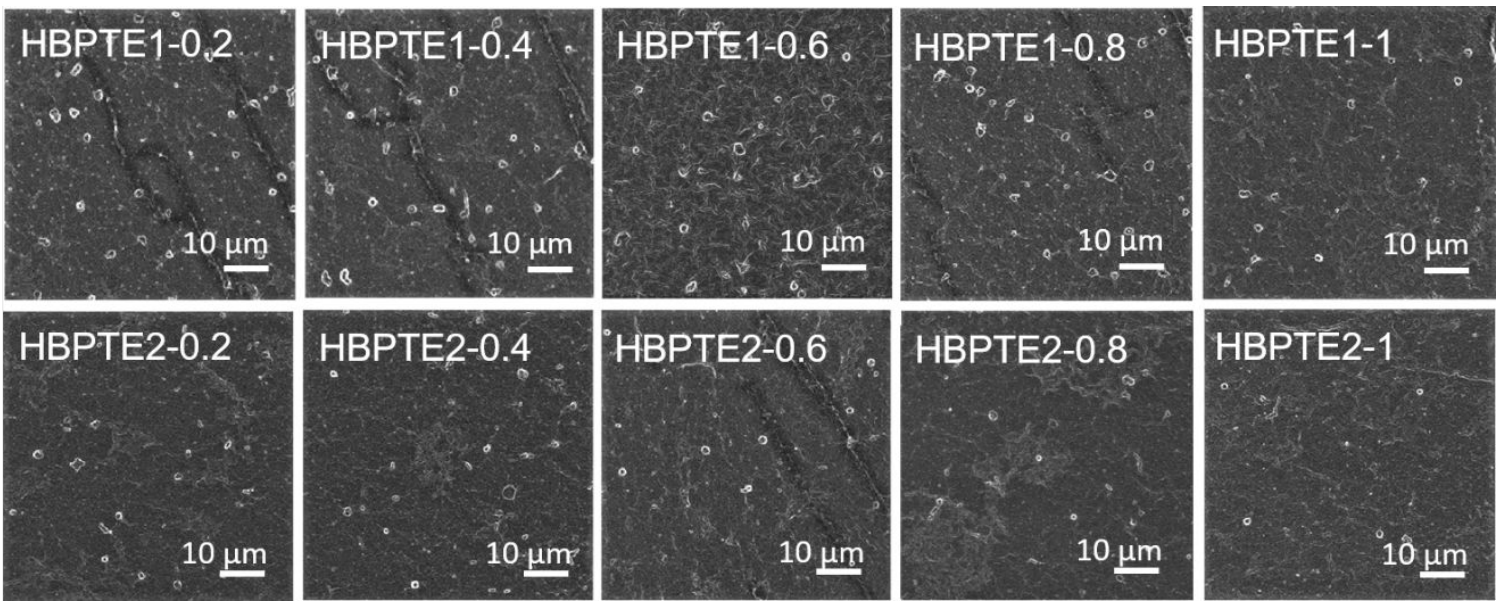

Figure S17: SEM images of adhered platelets on cured HBPTE glue. 